\title{
Multi-ion Mach-Zehnder interferometer with artificial nonlinear interactions
}

\author{
Y. M. $\mathrm{Hu}^{1,2,4}$, W. L. Yang ${ }^{1}$, X. Xiao ${ }^{3}$, M. Feng ${ }^{1},{ }^{*}$ and C. Lee ${ }^{4 \dagger}$ \\ ${ }^{1}$ State Key Laboratory of Magnetic Resonance and Atomic and Molecular Physics, \\ Wuhan Institute of Physics and Mathematics, Chinese Academy of Sciences, \\ and Wuhan National Laboratory for Optoelectronics, Wuhan 430071, China \\ ${ }^{2}$ Graduate School of the Chinese Academy of Sciences, Beijing 100049, China \\ ${ }^{3}$ College of Physics and Information Science, Hunan Normal University, Changsha 410081, China and \\ ${ }^{4}$ State Key Laboratory of Optoelectronic Materials and Technologies, \\ School of Physics and Engineering, Sun Yat-Sen University, Guangzhou 510275, China
}

\begin{abstract}
We show how to implement a Mach-Zehnder interferometry based upon a string of trapped ions with artificial nonlinear interactions. By adiabatically sweeping down/up the coupling strength between two involved internal states of the ions, we could achieve the beam splitting/recombination. Based on current techniques for manipulating trapped ions, we discuss the experimental feasibility of our scheme and analyze some undesired uncertainty under realistic experimental environment.
\end{abstract}

PACS numbers: 42.50.Dv, 06.30.Ft, 03.67.Ac

\section{INTRODUCTION}

Due to long coherence time of some specific hyperfine states, high controllability of operations and high efficiency of state detection, the trapped atomic ion system has been considered as a promising candidate for quantum information processing. In particular, since they could be nearly perfectly prepared in some entangled states, the trapped ions have attracted considerable attention for high-precision quantum metrology $[1-5]$.

It is generally believed that the measurement precision can be enhanced from the standard quantum limit (SQL) or shot noise limit to the Heisenberg limit by utilizing multipartite entanglement [6]. For example, it has demonstrated that the measurement precision may reach the Heisenberg limit by using a NOON state $\left(|N\rangle_{a}|0\rangle_{b}+|0\rangle_{a}|N\rangle_{b}\right) / \sqrt{2}$, which is a superposition of $\mathrm{N}$ particles in mode $a$ with zero particle in mode $b$ and vice versa. However, most of the relevant proposals on high-precision interferometry of trapped ions are subject to limited numbers of ions or to the requirement for individual addressing of ions, which restricts the scalability of those interferometry schemes.

In this article, we consider a Mach-Zehnder (MZ) interferometry using large numbers of entangled trapped ions. The MZ interferometry consists of a beam splitter for splitting the incoming state and another beam splitter for state recombination. It has been shown that the adiabatic MZ interferometry is an optimal candidate for high-precision measurement $[7,8]$. To achieve such an interferometry with trapped ions, we have to realize a nonlinear giant-spin Hamiltonian with anisotropic interaction. The key point is how to generate the nonlinear interactions and then achieve the beam splitting and recombination. By applying a pair of lasers to manip-

\footnotetext{
*Corresponding author. Email: mangfeng@wipm.ac.cn

${ }^{\dagger}$ Corresponding author. Email: chleecn@gmail.com
}

ulate independently their center-of-mass (COM) modes (either transverse or longitudinal vibrational modes), the nonlinear giant-spin Hamiltonian with anisotropic interaction could be simulated bases upon an array of trapped ions in a linear trap. By adjusting the coupling strength (i.e. the Rabi frequency) between the two involved hyperfine states, the MZ interferometry could be carried out adiabatically. With current techniques for manipulating trapped ions, we discuss the experimental feasibility of our scheme and the difficulty due to decoherence in realistic experimental environment.

The paper is structured as follows. In the next section, we describe the physical system and derive its effective Hamiltonian. In Sec. III, we show how to realize the MZ interferometer by using quantum adiabatic processes through dynamical bifurcations. Then, we discuss the experimental feasibility in Sec. IV and analyze the undesired uncertainty due to decoherence in Sec. V. In the last section, we briefly summarize our results.

\section{PHYSICAL SYSTEM AND ITS EFFECTIVE HAMILTONIAN}

We consider $\mathrm{N}$ identical ions confined in a linear trap, as shown in Fig. 1, where the three ionic states under our consideration are denoted by two hyperfine ground spin states $(|\downarrow\rangle,|\uparrow\rangle)$ and an excited state $|e\rangle$, respectively. In our scheme, all the ions are irradiated simultaneously by two traveling-wave laser beams with different frequencies, which coherently couple the hyperfine ground states through the optically stimulated Raman transitions by adiabatically eliminating the excited state $|e\rangle$. Here we assume the detuning of the optical fields from the electronic resonance to be much larger than the excited state linewidth and the corresponding coupling strength. As indicated in Fig. 1(a), under an usual rotating wave approximation to the laser frequencies, the interaction Hamiltonian between the fields and the ions is written as 

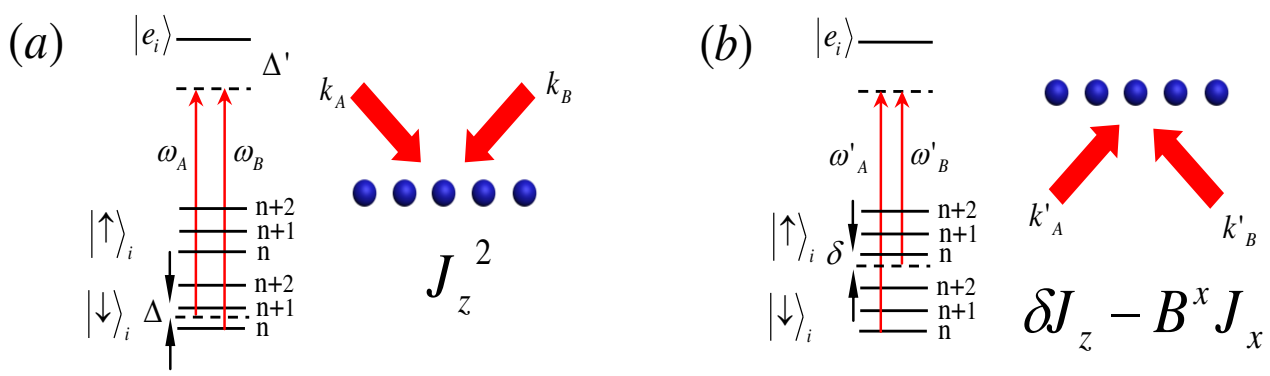

FIG. 1: (color online) Laser coupling scheme for simulating the Hamiltonian (2). (a) Physical realization of the nonlinear term of $\mathrm{J}_{z}^{2}$ : a Stark shift regarding the level $|\downarrow\rangle_{i}$ is created if two laser beams uniformly radiate the ions for the excitation from $|\downarrow\rangle_{i}$ to $|e\rangle_{i}$ with a large detuning $\Delta^{\prime}$. (b) Physical realization of the terms of $\mathrm{J}_{x}$ and $\mathrm{J}_{z}$ : two additional laser beams are used to induce the carrier transitions corresponding to the term of $\mathrm{J}_{x}$; and therefore the detuning to the resonant transition $\delta=\omega_{0}-\left(\omega_{A}^{\prime}-\omega_{B}^{\prime}\right)$ will generate the longitudinal-field term $\delta J_{z}$. See more details in Appendix A.

$(\hbar=1)[9]$

$$
H_{1}=\nu a^{\dagger} a-\frac{1}{\sqrt{N}} \sum_{i=1}^{N} \Omega_{z} \eta_{z}\left[\left(a^{\dagger}+a\right) \sigma_{z}^{i} e^{-i(\nu-\Delta) t}+\text { h.c. }\right] \text {, }
$$

where $\sigma_{z}^{i}=|\downarrow\rangle_{i}\langle\downarrow|-| \uparrow\rangle_{i}\langle\uparrow|$ is the population inversion operator for the $i$-th ion, $a^{\dagger}(a)$ is the creation (annihilation) operator of the center-of-mass (COM) mode and $\Omega_{z}$ is the uniform effective Rabi frequency. $\nu$ is the vibrational frequency of the COM mode and $\Delta$ is the detuning from the vibrational frequency. $\eta_{z}$ denotes the LambDicke parameter $k_{z} \sqrt{\hbar / 2 M \nu}$ with the ion mass $M$ and the laser wavevector difference $k_{z}=k_{B, z}-k_{A, z}$.

By tuning frequency differences and radiation directions of the pair of lasers along with independent manipulation of transverse or longitudinal COM modes, we may obtain following effective Hamiltonian under some approximations and canonical transformations,

$$
H_{e f f}=\delta J_{z}-B^{x} J_{x}-\lambda J_{z}^{2},
$$

where the collective angular momentum for all the ions are defined as $J_{k}=\sum_{i=1}^{N} \sigma_{k}^{i} / 2$ with $k=(x, y, z)$, the longitudinal field $\delta$ is the internal energy gap between the two spin states $(|\downarrow\rangle,|\uparrow\rangle)$, and the transverse field $B^{x}$ corresponds to the effective Rabi frequency, and the effective spin-spin nonlinear interaction is given as $\lambda=$ $8 \Omega_{z}^{2} \eta_{z}^{2} /(N \Delta)$. More details of our derivation are given in Appendix A. These parameters $\delta, B^{x}$, and $\lambda$ could be controlled by Rabi frequencies, wavevector differences of the lasers and the detunings.

\section{MZ INTERFEROMETRY VIA ADIABATIC OPERATIONS}

The MZ interferometry consists of a beam splitter for splitting input states and another beam splitter for recombining output states. Based upon the trapped-ion system (2), the two beam splitters for a MZ interferometry could be achieved by adiabatic quantum evolution through dynamical bifurcations.
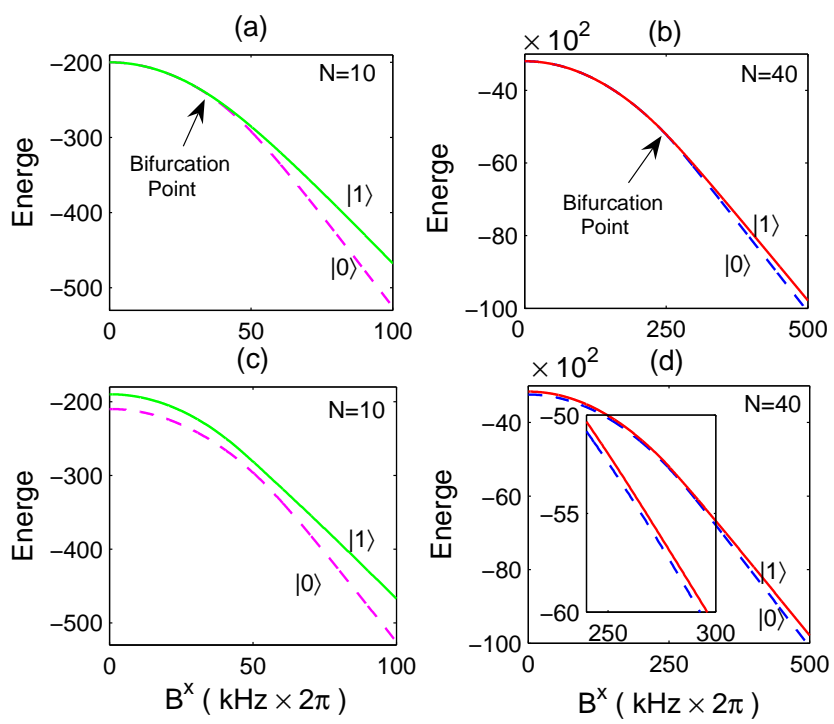

FIG. 2: (Color online) The energy spectra of the ground state $|0\rangle$ (dashed line) and the first excited state $|1\rangle$ (solid line) with respect to $B^{x}$ for different values of $\delta$ and $N$. If $\delta=0$, there is a bifurcation from degeneracy to non-degeneracy of the two lowest states $|0\rangle$ and $|1\rangle$ when $B^{x}$ increases, where (a) $\mathrm{N}=10$ and (b) $\mathrm{N}=40$. The beam splitting can be achieved by adiabatic passage through such a bifurcation. If $\delta \neq 0$, the degeneracy breaks down even in the weak limit of $B^{x}$, where $\delta=\lambda / 4$, with $\mathrm{N}=10$ (c) and $\mathrm{N}=40$ (d). The other parameters are chosen as $\eta_{z}=0.1, \Delta=0.01 \mathrm{MHz}, \Omega_{z}=2 \pi \times 100 \mathrm{kHz}$ $(N=10)$ and $2 \pi \times 200 \mathrm{kHz}(N=40)$.

In the collective spin representation, the trapped-ion system is regarded as an ensemble of $N$ spin- $1 / 2$ particles. Let $|j m\rangle$ stand for the joint eigenstate of the $\mathrm{SU}(2)$ Casimir operators $J^{2}$ and $J_{z}$, which satisfy the relations

$$
\begin{array}{r}
J^{2}|j m\rangle=j(j+1)|j m\rangle, \\
J_{z}|j m\rangle=m|j m\rangle,
\end{array}
$$

with $j=\frac{N}{2}$ and $m=\frac{N}{2}, \frac{N}{2}-1, \ldots,-\frac{N}{2}$ [10]. $\left|\frac{N}{2},-\frac{N}{2}\right\rangle$ $\left(\left|\frac{N}{2}, \frac{N}{2}\right\rangle\right)$ means all ions in the hyperfine state $|\downarrow\rangle(|\uparrow\rangle)$. 
We first consider the case $\delta=0$. In the strong coupling limit $B^{x} \gg|\lambda|$, the ground state $|0\rangle$ and the first excited state $|1\rangle$ for the Hamiltonian $H_{\text {eff }}$ are non-degenerated, where $|0\rangle=\exp \left(i \frac{\pi}{2} J_{y}\right)\left|j=\frac{N}{2}, m=\frac{N}{2}\right\rangle$ is a spin coherent state, and $|1\rangle$ is a superposition of different states $|j m\rangle$. When we adiabatically tune $B^{x}$ from the strong coupling limit $\left(B^{x} \gg|\lambda|\right)$ to the weak coupling limit $\left(B^{x} \ll|\lambda|\right)$, the ground state $|0\rangle$ and the first excited state $|1\rangle$ evolve into the states $\left|\frac{N}{2},-\frac{N}{2}\right\rangle$ and $\left|\frac{N}{2}, \frac{N}{2}\right\rangle$, respectively. If $B^{x}=0$, the two lowest states turn to be degenerated. So in such an adiabatic passage, there exists a transition from non-degeneracy to degeneracy between $|0\rangle$ and $|1\rangle$, which can be regarded as a bifurcation [11, 12], as shown in (a) and (b) of Fig. 2. Similar to a single-particle MZ interferometry, where the ground state $|0\rangle$ and the first exited state $|1\rangle$ are utilized as two paths, the two states of maximum spin $\left|\frac{N}{2},-\frac{N}{2}\right\rangle$ and $\left|\frac{N}{2}, \frac{N}{2}\right\rangle$ can also be used as two paths for an N-particle MZ interferometry. Therefore, the achievement of the maximally pathentangled state $\left|\Psi_{p}\right\rangle=\frac{1}{\sqrt{2}}\left(\left|\frac{N}{2}, \frac{N}{2}\right\rangle+\left|\frac{N}{2},-\frac{N}{2}\right\rangle\right)$ implies accomplishment of the beam splitting. Then we turn off the lasers for a duration $T$ so that the maximally pathentangled state $\left|\Psi_{p}\right\rangle$ evolves into

$$
\left|\Psi_{p}^{\prime}\right\rangle=\frac{1}{\sqrt{2}}\left(e^{-i \frac{N}{2} \omega_{0} T}\left|\frac{N}{2}, \frac{N}{2}\right\rangle+e^{i \frac{N}{2} \omega_{0} T}\left|\frac{N}{2},-\frac{N}{2}\right\rangle\right),
$$

where $\omega_{0}$ is the frequency difference between the two hyperfine spin states $|\downarrow\rangle$ and $|\uparrow\rangle$.

There are two different methods for extracting the relative phase information between the two paths of the $\mathrm{N}$-particle MZ interferometry. In the case of few trapped ions, this could be carried out using controlled-NOT gates $C N O T_{\text {rest }}^{1}$ with the first ion being the control qubit and the rest ions being the target ones, followed by a
Hadamard operation $\left(H_{1}\right)$ on the first ion [3]. The relative phase information can be obtained by measuring the population of the first ion in the state $|\downarrow\rangle$ or $|\uparrow\rangle$. Taking $N=5$ as an example, this individually addressing method can be summarized briefly as follows,

$$
\begin{aligned}
& \left|\Psi_{p}^{\prime}\right\rangle \stackrel{N=5}{\longrightarrow} \frac{1}{\sqrt{2}}\left(e^{-i \frac{5}{2} \omega_{0} T}|\uparrow \uparrow \uparrow \uparrow \uparrow\rangle+e^{i \frac{5}{2} \omega_{0} T}|\downarrow \downarrow \downarrow \downarrow \downarrow\rangle\right) \\
& \stackrel{C N O T_{\text {rest }}^{1}}{\longrightarrow} \frac{1}{\sqrt{2}}\left(e^{-i \frac{5}{2} \omega_{0} T}|\uparrow \downarrow \downarrow \downarrow \downarrow\rangle+e^{i \frac{5}{2} \omega_{0} T}|\downarrow \downarrow \downarrow \downarrow \downarrow\rangle\right) \\
& \stackrel{H_{1}}{\longrightarrow} \cos \left(\frac{5}{2} \omega_{0} T\right)|\downarrow \downarrow \downarrow \downarrow \downarrow\rangle+i \sin \left(\frac{5}{2} \omega_{0} T\right)|\uparrow \downarrow \downarrow \downarrow \downarrow\rangle .(4)
\end{aligned}
$$

However, for systems of large numbers of ions, individual addressing becomes challenging and it becomes very difficult to extract the relative phase with the controlledNOT gate $C N O T_{\text {rest }}^{1}$. If we apply the inverse process of the first beam splitter for recombing the two paths, the relatives phase information indeed could be transferred into the population information of the two lowest states for the limit of $B^{x} \gg \lambda$. However, it is not easy to directly distinguish these two lowest states. Fortunately, the two lowest states for symmetric $(\delta=0)$ and asymmetric $(\delta \neq 0)$ systems of $B^{x} \ll \lambda$ are almost identical and the two lowest states $\left(\left|\frac{N}{2},-\frac{N}{2}\right\rangle,\left|\frac{N}{2}, \frac{N}{2}\right\rangle\right)$ become non-degenerate for an asymmetric system $(0<\delta<\lambda / 4)$, see Fig. 2 (c) and (d). Therefore, to extract the relative phase, after applying the inverse process of the beam splitting process, we suddenly apply a proper nonzero $\delta$ $(0<\delta<\lambda / 4)$ and then adiabatically decrease $B^{x}$ from $B^{x} \gg \lambda$ to $B^{x}=0$. This recombination procedure for extracting the relative phase with two adiabatic processes could be summarized as,

$$
\begin{aligned}
& \left|\Psi_{p}^{\prime}\right\rangle \stackrel{\text { adiabatic process of } \delta=0, B^{x}=0 \rightarrow B^{x} \gg \lambda}{\longrightarrow} \frac{\cos \left(N \omega_{0} T / 2\right)|0\rangle-i \sin \left(N \omega_{0} T / 2\right)|1\rangle}{\sqrt{2}} \\
& \stackrel{\text { adiabatic process of } \delta \neq 0, B^{x} \gg \lambda \rightarrow B^{x}=0}{\longrightarrow} \frac{\cos \left(N \omega_{0} T / 2\right)\left|\frac{N}{2}, \frac{N}{2}\right\rangle-i \sin \left(N \omega_{0} T / 2\right)\left|\frac{N}{2},-\frac{N}{2}\right\rangle}{\sqrt{2}} .
\end{aligned}
$$

Obviously, by measuring the population with all the ions in the state $|\downarrow\rangle$ or $|\uparrow\rangle$, the relative phase between the two paths could be obtained.

\section{EXPERIMENTAL FEASIBILITY}

Our scheme is feasible with current ion trap technology. If we employ a line of trapped ${ }^{40} \mathrm{Ca}^{+}$, the states $|\downarrow\rangle,|\uparrow\rangle$ and $|e\rangle$ are denoted by $\mathrm{S}_{1 / 2}\left(m_{j}=-1 / 2\right)$, $\mathrm{S}_{1 / 2}\left(m_{j}=1 / 2\right)$, and $\mathrm{P}_{1 / 2}\left(m_{j}=-1 / 2\right)$ [13]. To adiabat- ically eliminate the ionic excited state $|e\rangle$, the detuning of the optical fields from electronic resonance should be much larger than the excited state linewidth and the corresponding coupling strength, e.g. $\Delta^{\prime}=2 \pi \times 20 \mathrm{GHz}$. On the other hand, generation of the nonlinear term $\lambda J_{z}^{2}$ in the Hamiltonian (2) requires that the detuning $\Delta$ should be much smaller than the frequency $\nu$ of the longitudinal COM mode. As a result, in our case we may take $\nu=1 \mathrm{MHz}$ and $\Delta=0.01 \nu$. Besides, the coherence time of the motional states has reached $100 \mathrm{~ms}$ [14]. To change the effective coupling $B^{x}$ adiabatically, we may set $B^{x}(t)=\alpha-\beta t$, where $\alpha$ and $\beta$ are nonnegative pa- 
rameters, for example, $\alpha=500 \mathrm{kHz}$ and $\beta=50 \mathrm{kHz} / \mathrm{ms}$ with $0 \leq t \leq 10 \mathrm{~ms}$ in the case of $N=40$. Consider the system in a free evolution by $T=5 \mathrm{~ms}$. After a duration for about $35 \mathrm{~ms}$ including three adiabatic processes plus the free evolution, we could obtain the relative phase by measuring the population of all the ions in state $\left|\frac{N}{2},-\frac{N}{2}\right\rangle$ or $\left|\frac{N}{2},+\frac{N}{2}\right\rangle$.

Higher measurement precision for the trapped-ion MZ interferometer requires more ions involved. With more ions involved, however, besides more attentions should be paid to larger coupling strength, we also need to consider the detrimental influence from decoherence. Fortunately, currently available technologies enable the effective coupling strength to be on the order of $\mathrm{MHz}$, which meets the requirement of our proposal. Therefore, we focus below on the influence from decoherence.

\section{ESTIMATION OF FREQUENCY UNCERTAINTY UNDER DECOHERENCE}

There are several sources of decoherence. Here, as an example, we only consider the depasing decoherence influence on the estimation of the ionic Zeeman splitting $\omega_{0}$. The maximally entangled state of $N$ trapped ions undertaking MZ interferometry decreases the measurement uncertainty of $\omega_{0}$ by $\sqrt{N}$ times compared to the trapped ions in product states $[3,5]$. If decoherence due to environment is involved, the measurement precision would be seriously affected $[15,16]$ because entangled states are more sensitive to decoherence than product states. A recent experiment [17] has reported that the NOON state with 8 to 14 trapped ions subjects to correlated dephasing noise mostly because of magnetic field fluctuation and shows super-decoherence, where the coherence decays quadratically with the number of qubits. This correlated dephasing could be modeled by the Lindblad equation in the free evolution,

$$
\dot{\rho}=-i \omega_{0}\left[J_{z}, \rho\right]+\gamma\left(2 J_{z} \rho J_{z}-J_{z}^{2} \rho-\rho J_{z}^{2}\right)
$$

where $\gamma$ is the correlated dephasing rate, which is $N^{2} \gamma_{0}$ with $\gamma_{0}$ the dephasing rate of a single qubit. Here, we consider $\gamma_{0}=\gamma_{0}^{\prime} \Omega_{z}^{2} / \Delta^{\prime 2}$ to be the effective two-level dephasing rate [18] due to the two-photon Raman process, where $\gamma_{0}^{\prime}$ is the spontaneous decay rate of the excited state $|e\rangle$ for a single qubit. The uncertainty $\Delta \omega / \omega_{0}$ shown in Fig. 3 is calculated in the equivalent spin representation, where the details are given in Appendix B.

Repeating the implementation of our proposal by one hundred times, we find that the correlated dephasing seriously spoils the measurement precision of the entangled state, yielding the precision even lower than the SQL. By setting $\gamma_{0}^{\prime} \approx 2 \pi \times 20 \mathrm{MHz}, \Omega_{z}=2 \pi \times 200 \mathrm{kHz}$, and $\Delta^{\prime}=2 \pi \times 20 \mathrm{GHz}$, and considering free evolution by $T=5 \mathrm{~ms}$ in each implementation, we have $\gamma T=0.1$ and the uncertainty of $\omega_{0}$ in the case of three-ion entanglement is larger than the SQL, see Fig. 3. In order to enhance the precision, we have to reduce $\gamma$. A possible way is to enlarge $\Delta^{\prime}$ by ten times, which leads to $\gamma T$ to be about 0.001 , and thereby the measurement precision would approach the Heisenberg limit. Further improvement could be made by using refocusing pulses during the adiabatic operations to suppress the decay from $|e\rangle$.

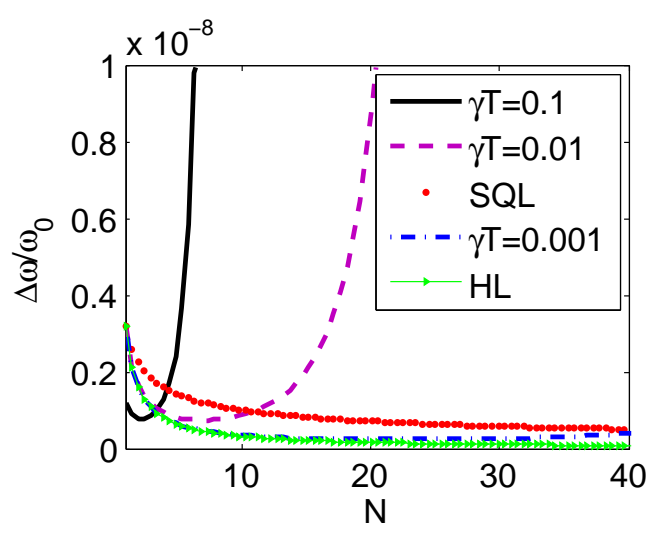

FIG. 3: (color online) The frequency uncertainty $\Delta \omega / \omega_{0}$ verse the total number of ions $N$. The dotted curve represents the estimated uncertainty in SQL corresponding to the product state, and the dash-dotted curve is the Heisenberg limit (HL) with the NOON state. We take $T=5 \mathrm{~ms}$ and $\omega_{0} \approx 2 \pi \times 3$ $\mathrm{GHz}$ at the magnetic field $B=0.1$ Tesla.

\section{CONCLUSIONS}

In summary, we have shown how to adiabatically carry out a MZ interferometer based upon multiple trapped ions with artificial nonlinear interactions. Influence from decoherence has also been discussed in our treatment. Since our interferometry proposal involves large numbers of trapped ions and works by global operations, we argue that the proposal would be useful for high-precision quantum metrology with trapped ions toward the Heisenberg limit [19].

We should also emphasize that, although our discussion above has only focused on the case of $\Delta>0$ and $\lambda>$ 0 for a trapped-ion MZ interferometer, our model enables to study spin squeezing in an ion trap by setting $\Delta<0$ for $\lambda<0$, which could be done by changing the directions of the laser beams $k_{A}^{\prime}$ and $k_{B}^{\prime}$ to make $B^{x}<0$ [20]. Our model also enables to investigate interaction blockade of spin flip with trapped ions for weak $B^{x}[21]$.

\section{ACKNOWLEDGMENTS}

This work is supported by the NBRPC under Grants No. 2012CB821305 and 2012CB922102, the NNSFC under Grants No. 10974225, 11075223 and 11004226, the NCETPC under Grant No. NCET-10-0850 and the 
Fundamental Research Funds for Central Universities of China.

\section{Appendix A: Generation of the effective Hamiltonian}

Below, we show how to generate the effective Hamiltonian (2) in Section II.

First, we discuss how to simulate the nonlinear term $\lambda J_{z}^{2}$ in the Hamiltonian $H_{e f f}$. As indicated in Fig. $1(\mathrm{a})$, irradiating the ions to the longitudinal COM mode by applying two non-copropagating laser beams with the wavevector difference $k_{z}$ along the $z$-axis, we have the Hamiltonian below in the rotating frame regarding $H_{0}=(\nu-\Delta) a^{\dagger} a$ under the rotating-wave approximation,

$H_{2}=e^{i H_{0} t} H_{1} e^{-i H_{0} t}=\Delta a^{\dagger} a-\frac{1}{\sqrt{N}} \sum_{i=1}^{N} \Omega_{z} \eta_{z}\left(a^{\dagger}+a\right) \sigma_{z}^{i}$.

Then applying a canonical transformation $e^{-S} H_{2} e^{S}$ with $S=\sum_{i=1}^{N} \frac{\Omega_{z} \eta_{z}}{\sqrt{N} \Delta}\left(a^{\dagger}-a\right) \sigma_{z}^{j}[22]$, we obtain

$$
H_{3}=\Delta a^{\dagger} a-\frac{2 \Omega_{z}^{2} \eta_{z}^{2}}{N \Delta} \sum_{i, j}^{N} \sigma_{z}^{i} \sigma_{z}^{j}
$$

Cooling the COM motion of the ions to its ground states, we obtain an effective spin-spin interaction in the Hamiltonian,

$$
\tilde{H}_{3}=-\lambda J_{z}^{2}
$$

with $\lambda=8 \Omega_{z}^{2} \eta_{z}^{2} /(N \Delta)$ and $J_{z}=\sum_{i=1}^{N} \sigma_{z}^{i} / 2$.

The generation of the transverse-field term $-B^{x} J_{x}$ requires additional lasers. As shown in Fig. 1(b), we set $\omega_{A}^{\prime}-\omega_{B}^{\prime}=\omega_{0}$, implying a resonant Raman process with respect to the two ground spin states of the ions. Such operations yield the transverse-field term [22]

$$
\tilde{H}_{2}=-B^{x} \sum_{i=1}^{N} \sigma_{x}^{i} / 2=-B^{x} J_{x}
$$

with $B^{x}$ an effective Rabi frequency $[22,23]$ and $J_{x}=$ $\sum_{i=1}^{N} \sigma_{x}^{i} / 2$.

If the Raman beams for generating the transverse-field term $\left(-B^{x} J_{x}\right)$ are non-resonant, i.e. the frequency difference of the two lasers $\omega_{A}^{\prime \prime}-\omega_{B}^{\prime \prime}=\omega_{0}-\delta$ with the detuning $\delta$, one can obtain the longitudinal-field term,

$$
\tilde{H}_{1}=\delta \sum_{i=1}^{N} \sigma_{z}^{i} / 2=\delta J_{z}
$$

see the schematic diagram Fig. 1(b).

Since the manipulation on the transverse COM mode is independent of the longitudinal COM mode, the total effective Hamiltonian reads as

$$
H_{e f f}=\tilde{H}_{1}+\tilde{H}_{2}+\tilde{H}_{3}=\delta J_{z}-B^{x} J_{x}-\lambda J_{z}^{2} .
$$

\section{Appendix B: Calculation of $\Delta \omega$}

For simplicity, we only consider the decoherence of the system during a free evolution. Suppose the system to be prepared in the maximally path-entangled state $\rho_{p}(0)=\left(\left|\frac{N}{2}, \frac{N}{2}\right\rangle+\left|\frac{N}{2},-\frac{N}{2}\right\rangle\right)\left(\left\langle\frac{N}{2}, \frac{N}{2}\right|+\left\langle\frac{N}{2},-\frac{N}{2}\right|\right) / 2$, where $\left|\frac{N}{2}, \frac{N}{2}\right\rangle$ and $\left|\frac{N}{2},-\frac{N}{2}\right\rangle$ stand for all ions in $|\uparrow\rangle$ and $|\downarrow\rangle$, respectively. After a time duration of $\mathrm{T}$, the system evolves into

$$
\begin{aligned}
\rho_{p}\left(\omega_{0}, T\right)= & \frac{1}{2}\left(\left|\frac{N}{2}, \frac{N}{2}\right\rangle\left\langle\frac{N}{2}, \frac{N}{2}\right|\right. \\
& +\left|\frac{N}{2},-\frac{N}{2}\right\rangle\left\langle\frac{N}{2},-\frac{N}{2}\right| \\
& +e^{-\gamma T N^{2}+i \omega_{0} T N}\left|\frac{N}{2},-\frac{N}{2}\right\rangle\left\langle\frac{N}{2}, \frac{N}{2}\right| \\
& \left.+e^{-\gamma T N^{2}-i \omega_{0} T N}\left|\frac{N}{2}, \frac{N}{2}\right\rangle\left\langle\frac{N}{2},-\frac{N}{2}\right|\right) .
\end{aligned}
$$

According to [16], the quantum Fisher information is given as

$$
F_{Q}=\operatorname{Tr}\left[\rho_{p}\left(\omega_{0}, T\right) A^{2}\right]=N^{2} T^{2} e^{-2 \gamma T N^{2}}
$$

where the Hermitian operator $A$ is the "symmetric logarithmic derivative" with its matrix elements defined as $A_{i j}=2\left[\rho_{p}^{\prime}\left(\omega_{0}, T\right)\right]_{i j} /\left(p_{i}+p_{j}\right), \quad \rho_{p}^{\prime}\left(\omega_{0}, T\right)=$ $\partial \rho_{p}\left(\omega_{0}, T\right) / \partial T$, with $p_{i}$ and $p_{j}$ the eigenvalues of $\rho_{p}\left(\omega_{0}, T\right)$, conditional on $p_{i}+p_{j}=0$ and $A_{i j}=0$.

The frequency uncertainty $\Delta \omega$ satisfies the quantum Cramér-Rao bound, [24]

$$
\Delta \omega \geq \frac{1}{\sqrt{k F_{Q}}}=\frac{1}{\sqrt{k} N T e^{-\gamma T N^{2}}}
$$

in the measurements on a set of $k$ probes.
[1] Huesmann R, Balzer Ch, Courteille Ph, Neuhauser W, and Toschek P E 1999 Phys. Rev. Lett. 821611

[2] Leibfried D, DeMarco B, Meyer V, Rowe M, Ben-Kish A, Britton J, Itano W M, Jelenković B, Langer C, Rosen- band T, and Wineland D J 2002 Phys. Rev. Lett. 89 247901

[3] Bollinger J J, Itano W M, Wineland D J, and Heinzen D J 1996 Phys. Rev. A 54 R4649 
[4] Sackett C A, Kielpinski D, King B E, Langer C, Meyer V, Myatt C J, Rowe M, Turchette Q A, Itano W M, Wineland D J, and Monroe C 2000 Nature (London) 404 256

[5] Leibfried D, Barrett M D, Schaetz T, Britton J, Chiaverini J, Itano W M, Jost J D, Langer C, and Wineland D 2004 Science 3041476

[6] Giovannetti V, Lloyd S, and Maccone L 2004 Science 306 1330

Giovannetti V, Lloyd S, and Maccone L 2006 Phys. Rev. Lett. 96010401

[7] Lee C 2006 Phys. Rev. Lett. 97150402

[8] Lee C, Huang J, Deng H, Dai H, and Xu J 2012 Front. Phys. 7109

[9] Lee P J, Brickman K -A, Deslauriers L, Haljan P C, Duan L-M, and Monroe C 2005 J. Opt. B: Quantum Semiclass. Opt. $7 \mathrm{~S} 371$

[10] Arecchi F T, Courtens E, Gilmore R, and Thomas H 1972 Phys. Rev. A 62211

Zhang W M, Feng D H, and Gilmore R 1990 Rev. Mod. Phys. 62867

[11] Lee C, Hai W, Shi L, and Gao K 2004 Phys. Rev. A 69 033611

Lee C 2009 Phys. Rev. Lett. 102070401

[12] Zibold T, Nicklas E, Gross C, and Oberthaler M K 2010 Phys. Rev. Lett. 105204101

[13] Home J P, McDonnell M J, Lucas D M, Imreh G, Keitch B C, Szwer D J, Thomas N R, Webster S C, Stacey D N, and Steane A M 2006 New J. Phys. 8188

[14] Kaler F S, Gulde S, Riebe M, Deuschle T, Kreuter A, Lancaster G, Becher C, Eschner J, H. Häffner, and R. Blatt 2003 J. Phys. B: At. Mol. Opt. Phys. 36623
[15] Shaji A and Caves C M 2007 Phys. Rev. A 76032111

[16] Dorner U, Demkowicz-Dobrzanski R, Smith B J, Lundeen J S, WasilewskiW, Banaszek K andWalmsley I A 2009 Phys. Rev. Lett. 102040403

Demkowicz-Dobrzanski R, Dorner U, Smith B J, Lundeen J S, Wasilewski W, Banaszek K, and Walmsley I A 2009 Phys. Rev. A $\mathbf{8 0} 013825$

[17] Monz T, Schindler P, Barreiro J T, Chwalla M, Nigg D, Coish W A, Harlander M, Hänsel W, Hennrich M, and Blatt R 2011 Phys. Rev. Lett. 106130506

[18] Pellizzari T 1997 Phys. Rev. Lett. 795242

[19] Wineland D J and Leibfried D 2011 Laser Phys. Lett. 8 175

[20] Law C K, Ng H T, and Leung P T 2001 Phys. Rev. A 63 055601

Ma J, Wang X, Sun C P, and Nori F quantumph/1011.2978.

[21] Lee C, Fu L -B, and Kivshar Y S 2008 EurophysLett. 81 60006

[22] Porras D and Cirac J I 2004 Phys. Rev. Lett. 92207901 Deng X -L, Porras D, and Cirac J I 2005 Phys. Rev. A $\mathbf{7 2} 063407$

[23] Friedenauer A, Schmitz H, Glueckert J T, Porras D, and Schaetz T 2008 Nat. Phys. 4757

[24] Braunstein S L and Caves C M 1994 Phys. Rev. Lett. 72 3439

Braunstein S L, Caves C M, and Milburn G J 1996 Ann. Phys. (NY) 247135

Helstrom C W 1976 Quantum Detection and Estimation Theory (New York: Academic) 